Diabetologe $2018 \cdot 14: 486-492$ https://doi.org/10.1007/s11428-018-0384-1 Online publiziert: 7. September 2018

(c) Der/die Autor(en) 2018

CrossMark

A. Jarasch' $\cdot$ A. Glaser $\cdot$ H. Häring ${ }^{1,2} \cdot$ M. Roden ${ }^{1,3} \cdot$ A. Schürmann ${ }^{1,4} \cdot$ M. Solimena $^{1,5} \cdot$ F. Theiss ${ }^{1,6} \cdot$ M. Tschöp ${ }^{1,7} \cdot$ G. Wess ${ }^{1,8} \cdot$ M. Hrabe de Angelis ${ }^{1,9}$

'Deutsches Zentrum für Diabetesforschung (DZD), Helmholtz Zentrum München, Neuherberg, Deutschland

${ }^{2}$ Institut für Diabetesforschung und Metabolische Erkrankungen des Helmholtz Zentrums München, Eberhard-Karls-Universität Tübingen, Tübingen, Deutschland

${ }^{3}$ Deutsches Diabetes Zentrum, Düsseldorf, Deutschland

${ }^{4}$ Deutsches Institut für Ernährungsforschung Potsdam-Rehbrücke, Nuthetal, Deutschland

${ }^{5}$ Paul Langerhans Institut des Helmholtz Zentrums München, Universitätsklinikum Carl Gustav Carus der TU Dresden, Dresden, Deutschland

${ }^{6}$ Institute for Computational Biology, Helmholtz Zentrum München - Deutsches Forschungszentrum für Gesundheit und Umwelt, Neuherberg, Deutschland

${ }^{7}$ Institute for Diabetes and Obesity, Helmholtz Zentrum München - Deutsches Forschungszentrum für Gesundheit und Umwelt, Neuherberg, Deutschland

${ }^{8}$ Helmholtz Zentrum München - Deutsches Forschungszentrum für Gesundheit und Umwelt, Neuherberg, Deutschland

${ }^{9}$ Institute of Experimental Genetics, Helmholtz Zentrum München - Deutsches Forschungszentrum für Gesundheit und Umwelt, Neuherberg, Deutschland

\title{
Mit Big Data zur personalisierten Diabetesprävention
}

Versorgung werden die Weichen dafür gestellt. Schon jetzt gibt es Systeme, die Krankheitsbilder analysieren und Diagnosen stellen. Forscher der „Mount Sinai School of Medicine“ stellten im Januar 2018 ein Forschungsergebnis vor, welches durch Anwendung künstlicher Intelligenz erhalten worden war. So kann durch Einsatz der intelligenten Software aus einem psychologischen Gespräch mit Jugendlichen mit einer Wahrscheinlichkeit von $82 \%$ erkannt werden, ob diese innerhalb der nächsten 2 Jahre an einer Psychose erkranken werden [1].

Einen Durchbruch auf dem Gebiet der Dermatologie gab es kürzlich: Die Klassifikation von Hautkrebs konnte durch den Einsatz künstlicher neuronaler Netze signifikant verbessert werden [2].

Laut den Analysten von „Price Waterhouse Coopers" (PwC) soll die künstliche Intelligenz in den nächsten Jahren Millionen Menschen zu besseren Therapien verhelfen und gleichzeitig dazu beitragen, die Gesundheitskosten signifikant zu senken. In der Medizin liegt ein sehr hohes Potenzial insbesondere in den beiden Bereichen bildgebende Diagnostik und Früherkennung von Krankheiten/ Pandemien [3].

\section{Diabetes - eine Volkskrankheit}

Für die Volkskrankheit Diabetes bietet die Digitalisierung vollkommen neue Möglichkeiten für eine bessere Prädiktion und Prävention. Dies ist dringend notwendig, denn die Zahl der Menschen mit Diabetes nimmt rapide zu. Weltweit vervierfachte sich diese seit 1980. Allein in Deutschland leiden knapp 7 Mio. Menschen daran, und jedes Jahr erkranken bis zu 500.000 neu an dieser Stoffwechselerkrankung. In Universitätskliniken liegt sogar bei $1 / 4$ aller Patienten ein manifester Diabetes vor [4].

》) Die Kosten für Therapie und Arbeitsausfälle durch Diabetes in Deutschland betragen $>16$ Mrd. $€ / J a h r$

Das Leiden sowie der Verlust an Lebensqualität der Betroffenen sind besonders in späteren Phasen der Erkrankung 
Hier steht eine Anzeige.

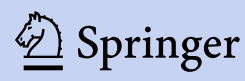




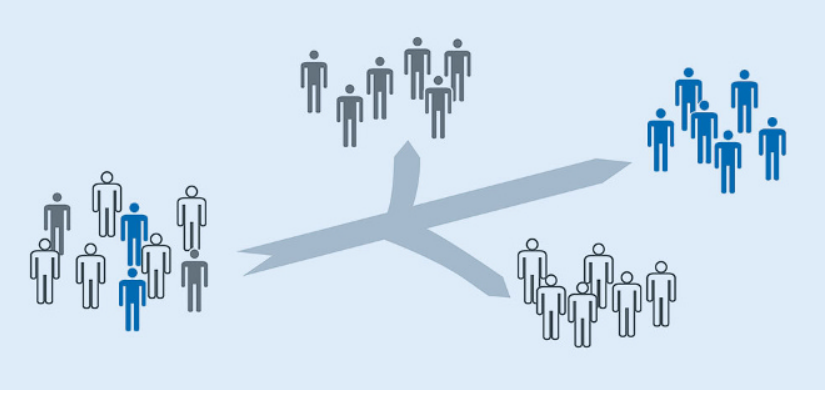

Abb. 1 ॥ Auftreten unterschiedlicher Subtypen (grau, dunkelblau, blassblau) des Prädiabetes und Diabetes mit individuell verschiedenem Ansprechen auf Lebensstiländerungen und Therapie (erste Gruppen bereits identifiziert). (Quelle: Deutsches Zentrum für Diabetesforschung e.V. [DZD])

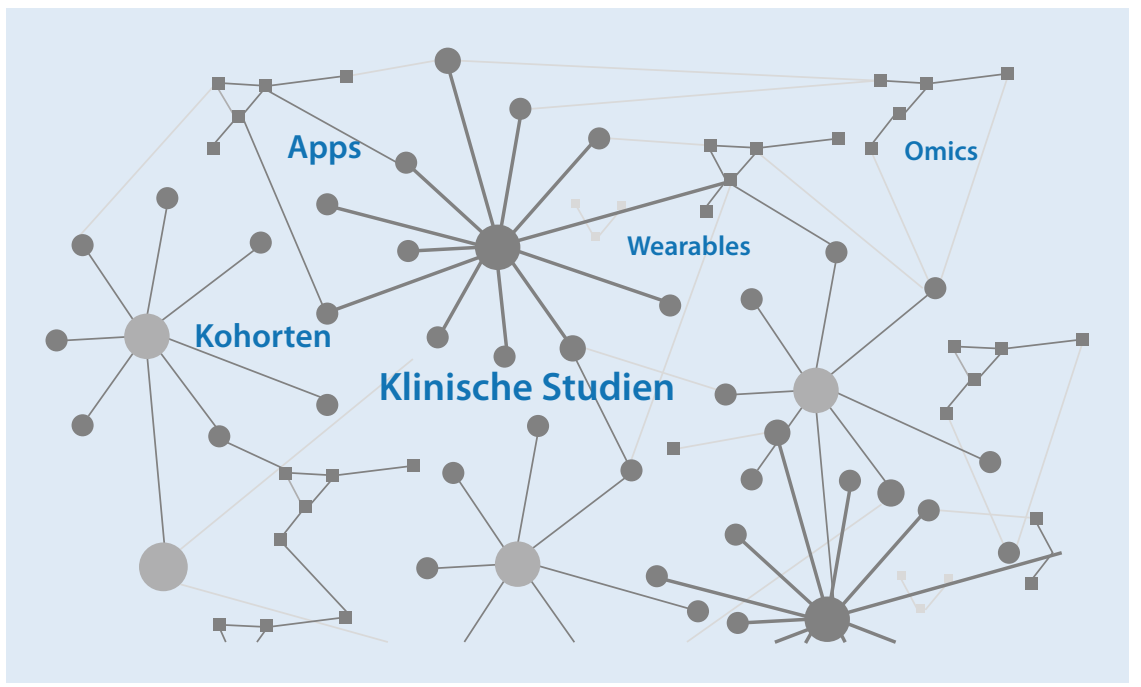

Abb. 2 \ Möglicher Gewinn neuer Erkenntnisse durch Verknüpfung unterschiedlicher Datenquellen und moderner Techniken der Informationsverarbeitung, KI künstliche Intelligenz. (Quelle: Deutsches Zentrum für Diabetesforschung e. V. [DZD])

groß. Auch belastet die Stoffwechselerkrankung zunehmend das deutsche Gesundheitssystem: Die Kosten für Therapie und Arbeitsausfälle belaufen sich auf mehr als 16 Mrd. $€$ im Jahr [5].

Diabetes ist eine komplexe Erkrankung, die durch ein vielschichtiges $\mathrm{Zu}$ sammenspiel von Genen, Lebensstil und Umweltfaktoren entsteht. Wissenschaftler des DZD (Deutsches Zentrum für Diabetesforschung) zeigten, dass es nicht „den“ Typ-2-Diabetes gibt, sondern unterschiedliche Subtypen sowohl des Präals auch des manifesten Diabetes, die auf Lebensstiländerungen und Therapie sehr individuell ansprechen [6]. Auch laut einer kürzlich in "The Lancet Diabetes \& Endocrinology" publizierten Arbeit existieren beimTyp-2-Diabetes weit komplexere Subtypen als bisher angenommen [7]. So lassen sich aus den großen schwe- dischen Kohorten 5 verschiedene Diabetesunterformen errechnen, die auch mit unterschiedlichen Outcomes korrelieren. Patienten von 3 dieser Subtypen sind mit Blick auf diabetische Folgeschäden als Hochrisikopersonen einzustufen. Bei den beiden weiteren Subtypen dagegen sind die Krankheitsverläufe weniger schwer. Auch eine DZD-Arbeitsgruppe identifizierte Subtypen mit einem sehr hohen Risiko für Organkomplikationen. Die hiervon Betroffenen haben eine ausgeprägte Insulinresistenz, eine niedrige Insulinsekretion und weisen auch eine Fettlebererkrankung auf - und das schon Jahre, bevor es mit einer sehr hohen Wahrscheinlichkeit zu einer manifesten Diabeteserkrankung kommt [8].

Nun gilt es, noch mehr solcher Untergruppen zu identifizieren und herauszufinden, welche davon jeweils welche Ei- genschaften aufweisen und wie Patienten mit dem betreffenden Subtyp behandelt werden müssten (• Abb. 1). Je kleiner und ausdifferenzierter diese Untergruppen werden, desto individueller und Erfolg versprechender werden die Prädiktion, Prävention oder Therapie ausfallen. Besonders für die Prävention ist es essenziell, frühe Prädiktoren zu finden, die bereits lange vor dem Ausbruch der Erkrankung ein sicheres Signal der Veränderungen im Körper widerspiegeln [9]. Das Auffinden solcher Prädiktoren sowie die damit verbundene Identifizierung von Untergruppen des Diabetes sind ein wesentlicher Schritt in Richtung personalisierte Medizin, die im Mittelpunkt der Forschungsaktivitäten des DZD steht.

\section{Prädiktion heute und morgen}

Hinsichtlich der Prädiktion können Erwachsene mit Hilfe des DIfE - Deutscher Diabetes-Risiko-Test ${ }^{\circledast} \quad$ (DRT; Bezugsquelle: DZD oder online: diabetesrisiko test.de) ihr persönliches Risiko ermitteln, innerhalb der nächsten 5 Jahre an einem Typ-2-Diabetes zu erkranken. Dem sehr aussagekräftigen und validierten Test liegen Daten großer epidemiologischer Studien zugrunde [10]. Damit er rechtzeitig durchgeführt wird, ist es wichtig, Menschen ohne Diabetes und besonders gefährdete Personen zu erreichen. Allerdings zeigte die jüngst vom Robert Koch-Institut (RKI) in Deutschland veröffentlichte Studie „Krankheitswissen und Informationsbedarfe - Diabetes mellitus", dass ein deutlicher Bedarf für eine zielgruppenspezifische Information und Kommunikation zum Thema Diabetes besteht und sich konkrete Wissenslücken u.a. zur Entstehung von Diabetes und Risikofaktoren in der Allgemeinbevölkerung identifizieren lassen [11]. Somit stellt sich die Frage, wie man die Digitalisierung, die zurzeit in allen Lebensbereichen unserer Gesellschaft Einzug hält, für ein besseres Bewusstsein („awareness") sowie eine bessere Prädiktion und Prävention nutzen kann.

Durch den Aufbau eines DDPC (digitales Diabetespräventionszentrum) soll unter Einbeziehung großer Bevölkerungsgruppen, Gesundheits- und Forschungsdaten aus unterschiedlichsten 
Quellen und innovativen Informationstechnologien die Chance genutzt werden, Subtypen des Diabetes in der Bevölkerung frühzeitig zu erkennen und eine zielgerichtete personalisierte Prädiktion und Prävention zu ermöglichen. Nur so können der Ausbruch der Stoffwechselerkrankung bzw. die Entstehung von Folgeerkrankungen eingedämmt oder verhindert werden, was letztendlich auch zu einer Kostensenkung im Gesundheitswesen führen wird. Dafür setzen sich das DZD und das Helmholtz Zentrum München ein. Patienten, die Daten zur Verfügung stellen, profitieren direkt. Sie erhalten diese mit einem besonderen Mehrwert zurück - einer speziell auf jeden Einzelnen zugeschnittenen personalisierten Prävention.

\section{Nutzung von Big Data}

\section{Einbezug bisher verborgener Daten}

Laut einer Umfrage des Digitalverbands Bitkom und der BTA (Bayrische TelemedAllianz) nutzen rund $45 \%$ der deutschen Smartphonebesitzer GesundheitsApps, um ihre Körper- und Fitnessdaten aufzuzeichnen. Gewünscht ist auch die Möglichkeit, die eigene Patientenakte digitalisieren zu können. So gaben $32 \%$ der Befragten an, bereits medizinische Untersuchungsergebnisse auf CD erhalten $\mathrm{zu}$ haben, und weitere $43 \%$ würden die Befunde künftig gerne in digitaler Form zur Verfügung gestellt bekommen [12].

\section{》) Millionen von Daten liegen im Verborgenen}

Auch bei den Krankenkassen liegen zahlreiche Daten vor, beispielsweise aus Disease-Management-Programmen (DMP). Das bedeutet: Ärzte, Krankenhäuser, Krankenkassen, Patienten, Gesundheitsinteressierte, das gesamte Gesundheitssystem sowie Forschungseinrichtungen verfügen insgesamt gesehen über einen riesigen Datenschatz: Millionen von Patientenakten, die jede für sich die Krankheitsgeschichte eines Menschen dokumentiert.

Diabetologe 2018 $\cdot 14: 486-492$ https://doi.org/10.1007/s11428-018-0384-1

(c) Der/die Autor(en) 2018

\author{
A. Jarasch · A. Glaser $\cdot$ H. Häring $\cdot$ M. Roden · A. Schürmann - M. Solimena $\cdot$ F. Theiss · \\ M. Tschöp - G. Wess · M. Hrabe de Angelis
}

\title{
Mit Big Data zur personalisierten Diabetesprävention
}

\section{Zusammenfassung}

Seit 1980 vervierfachte sich die Zahl der Menschen mit Diabetes weltweit. Allein in Deutschland leiden knapp 7 Mio. Menschen an dieser Stoffwechselerkrankung, und jedes Jahr erkranken bis zu 500.000 neu daran. Diese Zahlen machen deutlich, wie dringend neue wirksame Präventionsmaßnahmen und innovative Behandlungsformen benötigt werden. Die Digitalisierung ermöglicht es, die Volkskrankheit Diabetes in einer neuen Dimension zu erforschen, um sehr früh Subtypen dieser Stoffwechselerkrankung zu erkennen und geeignete personalisierte Präventionsmaßnahmen anzubieten. Mit dem Aufbau eines digitalen Diabetespräventionszentrums könnten Gesundheits- und
Forschungsdaten aus unterschiedlichsten Quellen zusammengeführt und mit innovativen Informationstechnologien (IT: Informationstechnik) analysiert und ausgewertet werden, um unterschiedliche Diabetessubtypen identifizieren und spezifische Präventions- und Therapiemaßnahmen anbieten zu können, die durch die enge Zusammenarbeit mit der Bevölkerung direkt einsetzbar wären.

\section{Schlüsselwörter}

Prädiabetes · Subtypen · Präventionsmedizin . Medizinische Informatik · Künstliche Intelligenz

\section{Big data for personalized diabetes prevention}

\section{Abstract}

Since 1980, the number of people with diabetes has quadrupled worldwide. In Germany alone, almost 7 million people suffer from this metabolic disease and every year, there are up to 500,000 new diagnoses. These numbers show the urgent need for new effective prevention measures and innovative forms of treatment. Digitalization makes it possible to explore the widespread disease of diabetes in a new dimension in order to identify subtypes of diabetes very early on and offer suitable personalized preventive measures. With the establishment of a Digital Diabetes Prevention Center, health and research data from a wide variety of sources could be brought together, analysed and evaluated using innovative information technology (IT) capabilities to identify different diabetes subtypes and offer specific prevention and therapy measures that can be used directly through close cooperation with the population.

\section{Keywords}

Prediabetic state $\cdot$ Subtypes $\cdot$ Preventive medicine $\cdot$ Medical informatics $\cdot$ Artificial intelligence
Neu ist jetzt, dass es mit den modernen Informationstechnologien möglich wäre, diese Daten zu vernetzen, Querverbindungen und Gemeinsamkeiten zu identifizieren und damit Erkenntnisse zu liefern, die bislang verborgen blieben, aber nun zum Wohle des Menschen eingesetzt werden könnten (• Abb. 2).

\section{Prävention durch Kombination von Big Data und bestehender Expertise}

Als öffentlich geförderte Forschungsinstitutionen wollen das DZD und das Helmholtz Zentrum München mit dem
DDPC die Bevölkerung in den Mittelpunkt der Aktivitäten setzen, um

- bei Personen, die ein Diabetesrisiko tragen, den Ausbruch der Erkrankung zu verhindern, und

- bei den bereits erkrankten Personen die Entwicklung der Spätfolgen zu stoppen.

Durch die Vernetzung von Big Data, der wissenschaftlichen Exzellenz in der Diabetesforschung und Datenverarbeitung sowie der klinischen Anbindung hat das DDPC beste Voraussetzungen, neue Wege $\mathrm{zu}$ beschreiten, um mittelfristig personalisierte Präventions- 


\section{Leitthema}

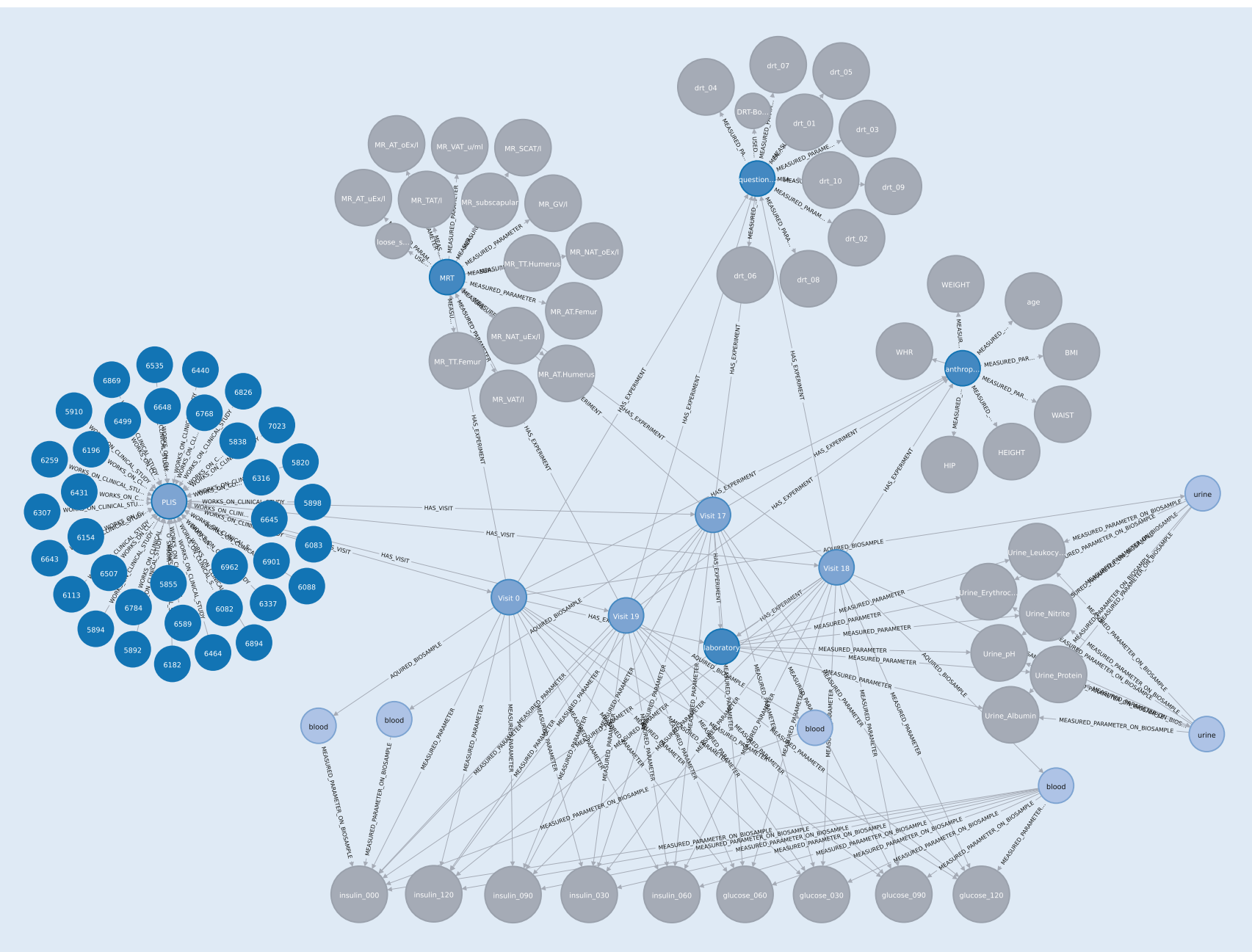

Abb. 3 A DZD CONNECT: Verknüpfung von Daten unterschiedlichster Quellen aus der Prädiabetes Lebensstil-Interventions-

Studie (PLIS) in einer Graphdatenbank. (Quelle: Deutsches Zentrum für Diabetesforschung e.V. [DZD])

und Therapiemöglichkeiten anzubieten. Neue Strukturen, neue Techniken, neue Algorithmen und Analyseverfahren sollen entwickelt und angewendet werden, um aus den Datenmengen verwertbares Wissen und belastbare Erkenntnisse zu ziehen.

\section{Verknüpfung von Daten unterschiedlichster Quellen}

Im DZD liegen bereits Daten aus unterschiedlichsten Quellen vor - wie aus klinischen Studien, Kohorten, präklinischen Modellen, Technologien des Bereichs "next generation -omics" und Biobanken. Nun kommen noch Daten zu epigenetischen Veränderungen des genetischen Codes hinzu.

Durch Omics-Ansätze gelang es bereits, verschiedene Protein- und Metabo- litenbiomarker zu identifizieren, mittels derer die Diagnose von Prädiabetes zukünftig vereinfacht werden könnte [13, 14].

\section{I) Menschen, die dem DDPC Daten zur Verfügung stellen, würden durch individuelle Betreuung profitieren}

Im Moment wird die Graphdatenbank DZD CONNECT aufgebaut, um einen einfachen Zugriff aufdie Daten zu ermöglichen (•Abb. 3). Ziel ist es, die vorhandenen Daten unterschiedlichster Quellen zugänglich, wiederverwertbar und übergreifend nutzbar zu machen. Um die Entstehung von Typ-2-Diabetes noch besser verstehen, weitere interessante Muster er- kennen und Subgruppen definieren zu können, ist es wichtig, wesentlich größere Datenmengen auch von gesunden Personen aus der Bevölkerung einzubeziehen.

Viele Menschen erfassen bereits heute eigene Gesundheitsdaten, etwa über Trackingarmbänder, Wearables oder Fitness-Apps. Bislang greifen v. a. große internationale IT- (Informationstechnologie) und Pharmafirmen auf die vielfältig erhobenen Gesundheitsdaten von Apps und Wearables zu. Damit die Forschung aber auch in Zukunft gleichberechtigt mit der Industrie agieren und als starker Partner kooperieren kann, benötigt sie ebenfalls einen Zugang zu diesen Daten. Das Besondere am DDPC wäre, dass die Menschen, die ihm ihre Daten zur Verfügung stellen, auch einen konkreten Mehrwert haben. Sie würden eine individuelle Dia- 


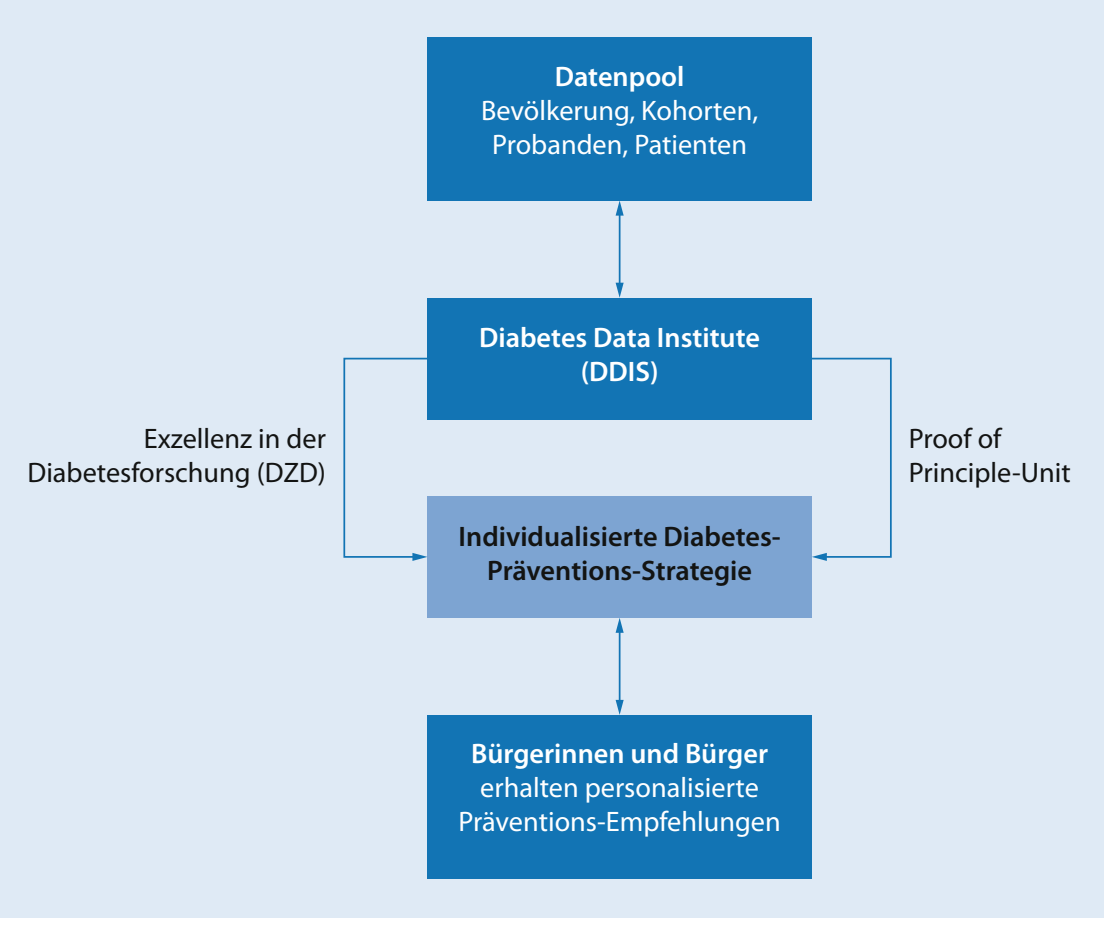

Abb. 4 \ Geplante Struktur des DDPC (digitales Diabetespräventionszentrum), DZD Deutsches Zentrum für Diabetesforschung. (Quelle: DZD/HMGU)

betestherapie bzw. -prävention erhalten, wissenschaftlich fundiert und frei von kommerziellen Interessenslagen.

Digitale Medien wie Apps können Personen zu für sie geeigneten Lebensstilmaßnahmen motivieren. Im DZD werden bereits erste Apps, insbesondere für Hochrisikogruppen, entwickelt und in Multicenterstudien getestet.

\section{Möglichkeiten zur Strukturierung des DDPC}

Das DDPC ist als nationale Initiative mit verschiedenen Standorten in Deutschland angedacht. Dabei bringt das Helmholtz Zentrum München die mathematische und informationstechnische Expertise ein, die klinische Expertise kommt u. a. vom DZD.

Diabetes-Data-Institute setzen sich aus Einheiten in den Bereichen Datenspeicherung, Datenplausibilität und Qualitätskontrolle, Algorithmenentwicklung sowie Data-Mining (unter Anwendung künstlicher Intelligenz) zusammen. Hier werden Daten aus unterschiedlichen Quellen wie klinischen Studien, Grundlagenforschung, Gesundheitsdaten der Probanden und

\section{Teilhabe der Bevölkerung am medizinischen Fortschritt}

Die Rolle der Bevölkerung wird sich im Rahmen der Digitalisierung maßgeblich ändern. Patientengruppen möchten bei der Testung neuer Systeme kooperieren. Von Patientenvertretern wird beklagt, dass man am medizinischen Fortschritt in Deutschland nicht vollumfänglich teilnehmen könne, da die Datenschutzbestimmungen Forschung und Innovation behindern [16]. Gemeinsam mit der Politik, den Fachgesellschaften und den Patientenverbänden müssen wir einen Weg finden, der den Zugang zu Daten auf sicherem und geschütztem Wege garantiert, aber andererseits den medizinischen Fortschritt in einer digitalisierten Welt nicht verhindert. Der von der DDG (Deutsche Diabetesgesellschaft) entwickelte "code of conduct digital health" bietet hierzu eine gute Grundlage [17]. Noch ist das DDPC eine Zukunftsvision. Es gilt, die Politik davon überzeugen, in ein solches Zentrum zu investieren. Angesichts von Gesundheitskosten im Diabetesbereich von mehr als $16 \mathrm{Mrd}$ € jährlich wäre das sicher eine gute Investition.

\section{Fazit für die Praxis} personalisierte Therapien und Präventionsansätze ableiten zu können.

Experten in "computational biology“ aus München wiesen in einem Pilotprojekt unter Anwendung künstlicher Intelligenz bereits nach, dass sich hiermit Retinopathien besser klassifizieren lassen. Diese Forschungsstrategie wird nun weiterverfolgt, um entsprechende Erkenntnisse in Form neuerer, besserer Therapien und individuell maßgeschneiderter Vorsorgekonzepte umzusetzen [15].

Die Diabetes-Data-Institute werden von klinischen Zentren („clinical hubs“) flankiert, welche auf bestehenden Aktivitäten und Kooperationen des DZD und weiteren Institutionen aufbauen. "Proof of principle studies" werden mit den Partnerinstitutionen des DZD durchgeführt (『Abb. 4).
- Digitalisierung, Big Data und AI ermöglichen es, die Prädiktion und Prävention des Diabetes in einer vollkommen neuen Dimension zu erforschen. schaften und Patientenverbänden müssen wir einen Weg finden, der den Zugang zu Daten auf sicherem und geschütztem Weg garantiert, aber andererseits den medizinischen Fortschritt in einer digitalisierten Welt nicht verhindert.

- Besonders für die Prävention ist es essenziell, frühe Prädiktoren zu finden, die bereits lange vor dem Ausbruch der Erkrankung ein sicheres Signal der Veränderung im Körper widerspiegeln.

- Durch Etablierung eines DDPC könnten Subtypen des Diabetes in der Bevölkerung frühzeitig erkannt und eine zielgerichtete personalisierte
- Gemeinsam mit Politik, Fachgesell- 


\section{Prädiktion und Prävention ermög- licht werden. \\ - Personen, die Daten zur Verfügung stellen, sollen direkt profitieren und erhalten ihre Daten mit einem besonderen Mehrwert zurück - einer speziell auf sie zugeschnittenen personalisierten Prävention.}

\section{Korrespondenzadresse}

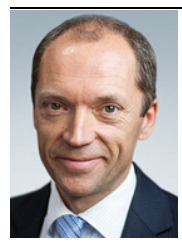

Prof. Dr. Dr. h.c. M. Hrabe de Angelis

Deutsches Zentrum für Diabetesforschung (DZD), Helmholtz Zentrum München Ingolstädter Landstr. 1, 85764 Neuherberg, Deutschland hrabe@helmholtzmuenchen.de

\section{Einhaltung ethischer Richtlinien}

Interessenkonflikt. A. Jarasch, A. Glaser, H. Häring, M. Roden, A. Schürmann, M. Solimena, F. Theiss, M. Tschöp, G. Wess und M. Hrabe de Angelis geben an, dass kein Interessenkonflikt besteht.

Dieser Beitrag beinhaltet keine von den Autoren durchgeführten Studien an Menschen oder Tieren.

Open Access Dieser Artikel wird unter der Creative Commons Namensnennung 4.0 International Lizenz (http://creativecommons.org/licenses/by/4.0/deed. de) veröffentlicht, welche die Nutzung, Vervielfältigung, Bearbeitung, Verbreitung und Wiedergabe in jeglichem Medium und Format erlaubt, sofern Sie den/die ursprünglichen Autor(en) und die Quelle ordnungsgemäßnennen, einen Link zur Creative Commons Lizenz beifügen und angeben, ob Änderungen vorgenommen wurden.

\section{Literatur}

1. Corcoran M et al (2018) Prediction of psychosis across protocols and risk cohorts using automated language analysis. World Psychiatry 17(1):67-75

2. Esteva A et al (2017) Dermatologist-level classification of skin cancer with deep neural networks. Nature 542:115-118

3. https://www.pwc.de/de/gesundheitswesenund-pharma/kuenstliche-intelligenz-revolutioniertdie-medizin.html. Zugegriffen:27. Juni 2018

4. Kufeldt J et al (2018) Prevalence and distribution of diabetes mellitus in a maximum care hospital: urgent need for HbA1c-screening. Exp Clin Endocrinol Diabetes 126(2):123-129

5. Jacobs E et al (2017) Healthcare costs of Type 2 diabetes in Germany. Diabet Med 34(6):855-861

6. Fritsche A et al (2018) Ergebnisse und Implikationen der PLIS Studie. DDG Kongress, 11.5.2018 (Vortrag)
7. Ahlvist E et al (2018) Novel subgroups of adult-onset diabetes and their association with outcomes: a data-driven cluster analysis of six variables. Lancet Diabetes Endocrinol 6(5):361-369

8. Stefan $\mathrm{N}$ et al (2016) Phenotypes of prediabetes and stratification of cardiometabolic risk. Lancet Diabetes Endocrinol 4(9):789-798

9. Liu X et al (2017) Quantifying critical states of complex diseases using single-sample dynamic network biomarkers. Plos Comput Biol 13(7):e1005633

10. Mühlenbruch Ketal (2014) Vorhersage des Risikos für Typ-2-Diabetes in der deutschen Bevölkerung mit dem aktualisierten DRT (DIfE - DEUTSCHER DIABETES-RISIKO-TEST). Ernährungsumschau 61(6):93

11. Paprott R et al (2018) Erste Ergebnisse der Studie „Krankheitswissen und Informationsbedarfe Diabetes mellitus. J Health Monit 3:3. https://doi. org/10.17886/RKI-GBE-2018-062

12. Bitkom-Umfrage: Fast jeder Zweite nutzt HealthApps. Ärzte Zeitung, 11. Mai 2017

13. von Toerne $C$ et al (2016) MASP1, THBS1, GPLD1 and ApoA-IV are novel biomarkers associated with prediabetes. Diabetologia 59(9):1882

14. Wang-Sattler R et al (2012) Novel biomarkers for pre-diabetes identified by metabolomics. Mol Syst Biol 8:615

15. Eulenberg P et al (2017) Reconstructing cell cycle and disease progression using deep learning. Nat Commun 8(1):463

16. Public Hearing Bundesministerin Wanka in Berlin ZukunftsForum „Gesundheit neu denken" 8. Sept. 2015

17. https://www.deutsche-diabetes-gesellschaft.de. Zugegriffen: 1. Juli 2018
Hier steht eine Anzeige. Springer 